\title{
CORRECTIONS TO CARRIER FREQUENCIES OF RADIO STATIONS \\ RBU AND GBR BASED ON STATE TIME AND FREQUENCY \\ STANDARD OF USSR
}

TABLE 1. Corrections to Carrier Frequencies of Radio Stations RBU and GBR Based on State Time and Frequency Standard of USSR

\begin{tabular}{|c|c|c|c|c|c|c|c|c|}
\hline \multicolumn{9}{|c|}{ December, 1972} \\
\hline Bate & $\begin{array}{l}\mathrm{RBU} 66 .(6) \mathrm{kHz} \\
\text { referred to } \\
21 \text { TU }\end{array}$ & $\begin{array}{l}\text { GBR } 16 \mathrm{kHz} \\
\text { referred to } \\
23^{6} \text { TU }\end{array}$ & Date & $\begin{array}{l}\text { RBU 66.(6) } \mathrm{kHz} \\
\text { referred to } \\
21 \text { hTU }\end{array}$ & $\begin{array}{l}\text { GBR } 16 \mathrm{kHz} \\
\text { referred to } \\
23 \mathrm{~T} \mathrm{~T}\end{array}$ & Date & \begin{tabular}{|l|}
$\mathrm{RBU} 66 .(6) \mathrm{kHz}$ \\
referred to \\
$21^{\mathrm{h}} \mathrm{TU}$
\end{tabular} & $\begin{array}{l}\text { GBR } 16 \mathrm{kHz} \\
\text { referred to } \\
23 \text { hT U }\end{array}$ \\
\hline $\begin{array}{r}1 \\
2 \\
3 \\
4 \\
5 \\
6 \\
7 \\
8 \\
9 \\
10\end{array}$ & $\begin{array}{l}-0,1 \cdot 10-10 \\
0,0 \\
-0,4 \\
-0,4 \\
=0,4 \\
=0,5 \\
-0,6 \\
-0,1 \\
+_{0}^{0}, 3\end{array}$ & $\begin{aligned}-0,4 \cdot 10-10 \\
-0,4 \\
+0,2 \\
+0,4 \\
+0,5 \\
+0,0 \\
-0,7 \\
-0,5 \\
0,0\end{aligned}$ & $\begin{array}{l}11 \\
12 \\
13 \\
14 \\
15 \\
16 \\
17 \\
18 \\
19 \\
20\end{array}$ & $\begin{array}{l}+0,4 \cdot 10-10 \\
+0,2 \\
+0,3 \\
-0,1 \\
=0,1 \\
=0,2 \\
=0,2 \\
-0,2 \\
0,0 \\
+0,1\end{array}$ & $\begin{array}{c}+0,6 \cdot 10-10 \\
-0,5 \\
0, .0 \\
0,0 \\
+0,5 \\
-0,1 \\
+0,5 \\
0.3\end{array}$ & $\begin{array}{l}21 \\
22 \\
23 \\
24 \\
25 \\
26 \\
27 \\
28 \\
29 \\
30 \\
31\end{array}$ & $\begin{array}{l}0,0 \cdot 10-10 \\
+0,1 \\
+0,1 \\
-0,1 \\
-0,2 \\
+0,2 \\
+0,1 \\
+0,4 \\
+0,3 \\
+0,1 \\
+0,2\end{array}$ & $\begin{array}{l}\quad 0,0 \cdot 10-10 \\
-0,3 \\
+0,4 \\
+0,3 \\
-0,4 \\
-0,5 \\
0.3 \\
+0,0 \\
-0,5 \\
-0,2\end{array}$ \\
\hline \multicolumn{8}{|c|}{ Average $0.0 \cdot 10^{-10}$} & $0.0 \cdot 10^{-10}$ \\
\hline
\end{tabular}

Translated from Izmeritel'naya Tekhnika, No. 3, p. 44, March, 1973.

(0) 1973 Consultants Bureau, a division of Plenum Publishing Corporation, 227 West 17th Street, New York, N.Y. 10011. All rights reserved. This article cannot be reproduced for any purpose whatsoever without permis sion of the publisher. A copy of this article is available from the rublisher for $\$ 15.00$. 